\title{
Fracture Nasal Bone: Causes, Presentation and Management in a Tertiary Care Center in Nepal
}

\author{
Rajeev K Mahaseth, Urmila Gurung, Narmaya Thapa, Bibhu Pradhan, Bijaya Kharel \\ Department of ENT- Head and Neck Surgery, Maharajgunj Medical Campus, Ganesh Man Singh Memorial \\ Academy of ENT- Head \& Neck Studies, Institute of Medicine, Kathmandu, Nepal
}

\section{Corresponding author:}

Urmila Gurung, MBBS, MS

Department of ENT- Head and Neck Surgery, Maharajgunj Medical Campus, Ganesh Man Singh Memorial Academy of ENT- Head \& Neck Studies, Institute of Medicine, Kathmandu, Nepal

Email:dr.urmila.gurung@gmail.com

Submitted : Mar 11, 2020

Accepted : Apr 13, 2020

\section{ABSTRACT}

\section{Introduction}

Fracture of nasal bone, the commonest facial fracture is frequently encountered in ENT practice. This study was conducted to assess the causes, presentation and management of fracture nasal bone in a tertiary care center.

\section{Methods}

A retrospective chart review was done of patients admitted from August 2017 to July 2019 for management of isolated nasal bone fracture in the department of ENT and Head and Neck Surgery, Tribhuvan University Teaching Hospital.

\section{Results}

Thirty-five patients ( 31 males and 4 females) of 13 to 86 years (mean age 26 years) underwent closed reduction of fracture nasal bone. The injury was sustained following physical assault in 13/35 (37\%), fall from height in 10/35 (29\%), road traffic accident in 8/35 (23\%) and sports injury in 4/35 (11\%). Pain, epistaxis, swelling over nasal dorsum, nasal deformity and wound were the presenting symptoms which most often occurred in combination. Close reduction was done under local anesthesia in 28/35 (80 \%) and under general anesthesia in $7 / 35$ (20\%). The time between trauma and closed reduction ranged from 1 to 16 days with a mean of 6.2 days.

\section{Conclusion}

Nasal bone fracture needing reduction was common following physical assault in males predominently of 21 to 30 years. A combination of pain, epistaxis and nasal deformity was the commonest presentation. Closed reduction of fracture under local anesthesia within 16 days of trauma was the usual practice.

Keywords: Closed reduction, facial trauma, local anesthesia, nasal bone fracture 


\section{INTRODUCTION}

T he nose is prone to trauma as it projects anteriorly in the center of the face. ${ }^{1,2}$ Fracture of nasal bone is the commonest facial fracture ${ }^{3}$ accounting upto $39 \%{ }^{4}$ and the third amongst all fractures of human skeleton.1,4 It could occur as isolated nasal bone fracture or be associated with multiple trauma. ${ }^{1,5}$ following physical assault, fall, accidents and sports injuries. 2,5,6

Quite often incase of non-displaced fracture, it may be treated conservatively but if associated with cosmetic and functional deformity, it needs to be treated surgically. ${ }^{7}$ Nasal bone fracture, if not treated timely, can be a challenge both aesthetically and functionally as it can lead to secondary nasal deformities ranging from 14 to $50 \%^{7}$ and chronic nasal obstruction. ${ }^{1,8}$ It is therefore important to be aware of the need for early referral and intervention in cases of nasal trauma. ${ }^{2}$ This study was conducted to assess the causes, presentation and management of nasal bone fracture in a tertiary care center. This will help in knowing the current scenario of this common condition in a tertiary care center.

\section{METHODS}

It was a retrospective study done after obtaining ethical clearance from the Institutional Review Committee. This study assessed the medical records of all patients admitted from August 2017 to July 2019 in the Department of ENT and Head and Neck Surgery, Tribhuvan University Teaching Hospital for management of isolated nasal bone fracture. Patients with nasal bone fracture in combination with maxillofacial trauma, head injury were excluded from this study. Those patients with non-displaced nasal bone fracture treated conservatively in the outpatient department were also excluded.

The age, gender, causes of nasal bone fracture, clinical presentations, any additional surgical procedures besides nasal bone fracture reduction, the type of anesthesia used, the interval between trauma and the procedure were noted from the medical records. The data were entered in Microsoft Excel (version 15). The mean, range and frequencies were calculated.

\section{RESULTS}

In the two-year period, 35 patients were admitted for closed reduction of the nasal bone fracture. Their age ranged from 13-86 years with the mean age being 28 (SD 13.5) years. The maximum patients i.e. 16 patients fell in the 21 to 30 years category. There were $31(88.5 \%)$ males and 4 (11.5\%) females.

The injury was sustained most frequently following physical assault in 13/35 (37\%) cases. Fall from
Table 1. Symptoms following nasal bone fracture

$$
(n=35)
$$

\begin{tabular}{lc}
\hline \multicolumn{1}{c}{ Symptoms } & Frequency \\
\hline Pain & $26(74.2 \%)$ \\
Epistaxis & $20(57.1 \%)$ \\
Swelling over nasal dorsum & $16(45.7 \%)$ \\
Nasal deformity & $14(40 \%)$ \\
Wound & $3(8.5 \%)$ \\
Combination of symptoms & \\
Pain, epistaxis and nasal deformity & $11(31.4 \%)$ \\
Pain, epistaxis and swelling over & \\
nasal dorsum & $7(20 \%)$ \\
Pain and swelling over nasal dorsum & $5(14.2 \%)$ \\
Pain and wound over nasal dorsum & $3(8.6 \%)$ \\
\hline
\end{tabular}

height 10/35 (29\%), road traffic accident 8/35 (23\%) and sports injury 4/35 (11\%) were the other causes leading to the injury.

Pain was the most common symptom followed by epistaxis, swelling over nasal dorsum and nasal deformity with wound being the least common symptom (Table 1). However, these symptoms occurred in combination in most of the instances rather than with only one presenting symptom (Table 1).

Of the 35 patients, 24 patients presented within 7 days whilst 10 presented between 8-14 days and one presented on $16^{\text {th }}$ day post trauma (Figure 1).

Seventeen patients got operated the same day of hospital admission, 14 on the next day, 3 after 2 days and one after 3 days of admission (Figure 2). The delay of 2 to 3 days from admission to procedure was due to unavailability of GA emergency slot at the earliest.

The time between trauma and the closed reduction of fracture ranged from 1 to 16 days with a mean of 6.2 days. All cases underwent reduction within 14

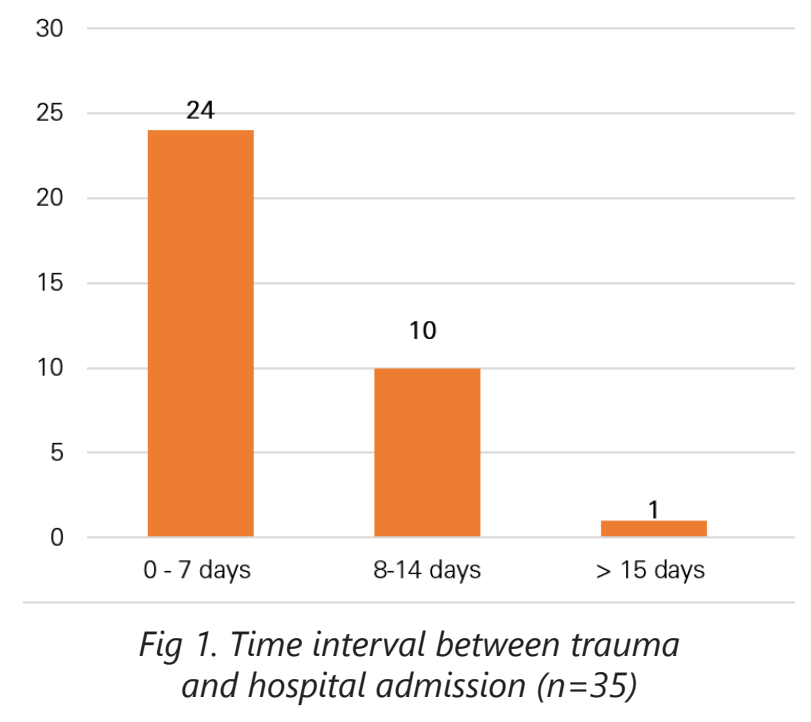




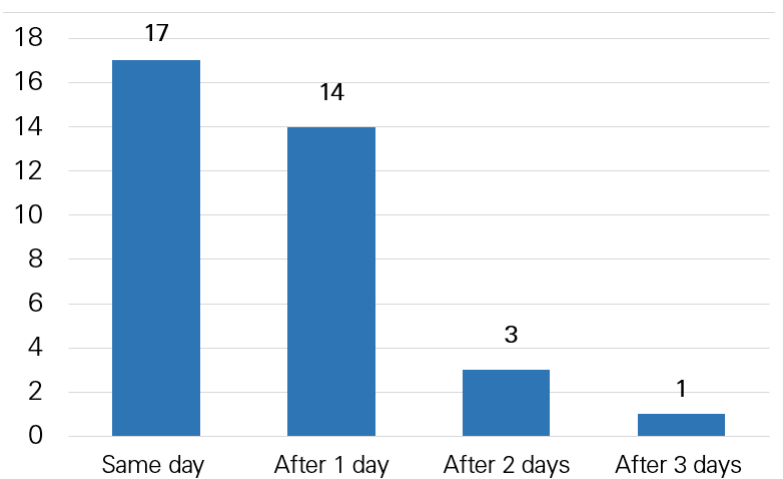

Fig 2. Time interval between hospital admission and closed reduction of fracture nasal bone $(n=35)$

days except one who had it done after 16 days of trauma (Figure 3).

Out of the 35 patients, $28(80 \%)$ had the reduction of nasal bone fracture done under local anesthesia (LA) whilst 7 (20\%) had it under general anesthesia (GA). Three patients underwent concurrent septoplasty along with reduction of fracture nasal bone in the same sitting of which two were under GA and one under LA. The lowest age of reduction under LA was 16.

Closed reduction was done under GA only incase of apprehensive patients or paediatric patients whenever GA was available in emergency slot. There were 4 and 3 patients respectively in the age range of 10 to 20 and 21 to 30 years who had closed reduction under general anesthesia.

\section{DISCUSSION}

Nasal bone fracture is one of the commonly presenting condition in the emergency department and ENT clinic. It can result from trauma to nose following physical assault, fall, accidents and sports injuries. ${ }^{2,5,6}$ In our study, physical assault was the commonest cause followed by fall, road traffic accident and sports injury. Byun et $\mathrm{al}^{4}$ found physical assault as the most common cause in general however fall was more common in females and sports injury in males. A systematic review by Hwang et al ${ }^{9}$ which analyzed 5561 patients showed physical assault as the leading cause equating to $36.3 \%$ followed by traffic accidents in $20.8 \%$, sports injury in $15.3 \%$ and fall injury in $13.4 \%$ in adults. In children, the most frequent causes were sports injury (59.3\%), physical assault (10.8\%), traffic accidents (8.3\%), collisions (5.0\%), and falls (3.3\%).

Nasal bone fracture usually is seen in younger age group. In our study, the age ranged from 13 to 86 years however the mean age was 26 years with most patients in the 21 to 30 years' age category. This was similar to a study done by Cil

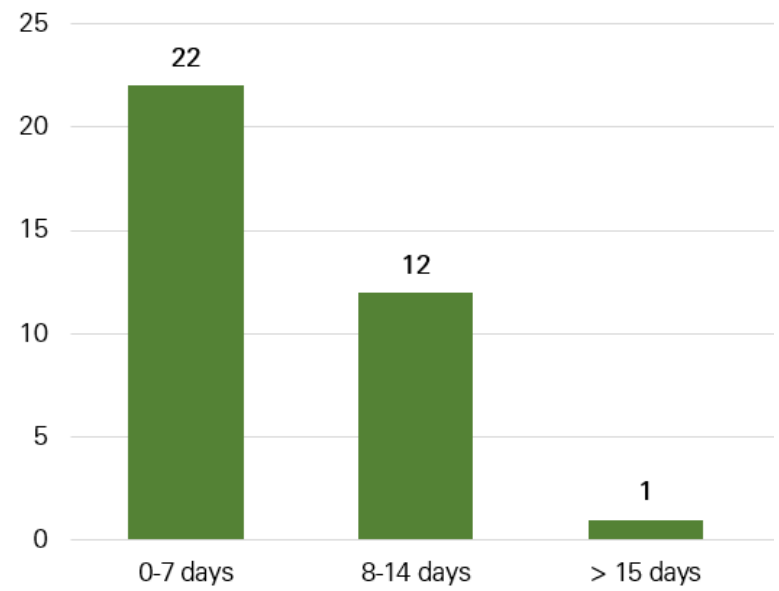

Fig 3. Time interval between trauma and closed reduction of fracture nasal bone $(n=35)$

et $\mathrm{a}^{5}$ where the mean age was 21 years amongst 45 patients whose age ranged from 6 to 32 years. Basheeth et $\mathrm{al}^{6}$ also noted a mean of 27 years on assessing 160 patients of 04 to 87 years presenting with nasal bone fracture in a referral centre. Male patients exceeded female patients in our study in concordance to other studies. ${ }^{2,4,5,6,10}$ The likeliness of young males being affected more could be because of their likeliness to be socially active, be engaged in outdoor activities and high risk jobs hence increasing the risk of trauma. ${ }^{4,5}$

Patient with nasal bone fracture can present with pain, swelling, external nasal deformity nasal blockage and epistaxis. ${ }^{2,5}$ They may not be the sole symptoms but present in combination, of which external nasal deformity can be the chief symptom. ${ }^{2}$ In our study, most patients had a combination of presenting symptoms comprising of pain, epistaxis and nasal deformity.

Nasal bone fracture is often detected clinically and further decision making is based on the clinical findings. ${ }^{1,6,14} \mathrm{X}$-ray of nasal bone has limited value in clinical decision making but can be an adjuvant for medicolegal purpose. ${ }^{5}$ In a study by Ridder et al ${ }^{14}$ out of the 187 patients assessed, 88 required no reduction inspite of $\mathrm{x}$-ray showing non dislocated fracture in 22 and dislocated fracture in 15 and contusion in 51 patients as their nasal cavity were patent and there was no external nasal deformity.

As per Mondin et al ${ }^{1}$ there are three major factors that could affect the outcome of nasal bone fracture management, namely the timing of the treatment, the anesthesia used (LA vs GA) and surgical technique (open vs closed).

Early reduction is possible if the deformity can be assessed before the oedema has set in. ${ }^{1}$ However, if there is soft tissue oedema, reassessment is needed in 3-4 days as oedema can mask the fracture ${ }^{1,2,11}$ and also cause difficulty in determining 
the adequacy of the reduction. ${ }^{11}$ In our study, all reduction except one was done within 14 days on an average of 6 days.

The ideal interval of treatment from trauma as emphasized by Staffel is within 2 weeks as reduction afterwards did not straighten the nose in his study..$^{12}$ Cil et al ${ }^{5}$ recommended reduction within 7 days of trauma for children and 10 days for adults. However, Basheeth et al ${ }^{6}$ advocated reduction upto 5 weeks as their study showed a good result of $77.5 \%$ for breathing and $85.6 \%$ for cosmesis for reduction upto 5 weeks. This is likely because bony callus takes $4-5$ weeks to form. ${ }^{6}$

Majority of the nasal bone fractures are managed by closed reduction with some needing second surgery at a later date. ${ }^{13}$ Closed reduction involves external digital manipulation with ocassional use of Walsham and Asch forceps to realign the bony fragments and/ or manipulation of depressed fragment using elevators intranasally. ${ }^{6,11,14}$ Concurrent septoplasty or submucosal resection is needed if associated with severe septal fracture. ${ }^{1,14}$ The incidence of post reduction deformities requiring septorhinoplasty was $22.5 \%$ in a study by Basheeth et al implicating traumatic edema, preexisting nasal deformity, and occult septal injury as the causes for acute reduction failures. ${ }^{6}$ Septorhinoplasty in the initial treatment of nasal bone fracture has been recommended in instances when patient desires cosmetic improvement without having to undergo second surgery. ${ }^{1,8}$ Three patients in our study needed septoplasty in the same sitting due to pre-existing septal deviation. Nasal packing and external nasal splinting have been used to stabilize and protect the reduction. ${ }^{1}$ All of our patients underwent nasal packing and external nasal splinting with their respective removal on second post-operative day and two weeks respectively.

The outcome of closed reduction under LA is comparable to GA in terms of functional and aesthetic outcome. ${ }^{714,15}$ In a prospective study by Waldron et al., ${ }^{15} 50$ consecutive patients each underwent reduction of simple fracture bone under general anesthesia and local anesthesia. 92\% of the patients tolerated the LA well. Acceptable cosmetic results were obtained in $86 \%$ in GA and $84 \%$ in LA. Watson et $\mathrm{al}^{7}$ in a randomized prospective study of 40 patients found comparable cosmetic and functional result in both LA and GA. Wild et al ${ }^{10}$ found $94 \%$ patients over 14 years of age preferred LA over GA. The discomfort associated with it was similar to that of a minor dental procedure. Given that reduction under LA has better safety profile, has lower cost, uses lesser hospital resources and still a has comparable result as GA. 10,11,15 Mondin et al ${ }^{1}$ recommended reduction of the fractured nasal bone under local anesthesia to be made a standard practice. General anesthesia could be indicated in certain circumstances like in pediatric patients, patients with severely depressed fractures needing open reduction, patient's personal preference and pain tolerance. 10,14

There are some limitations of our study. This study being a retrospective study, neither the short term or long term outcome of closed reduction of nasal bone fracture or the patient satisfaction could be assessed. A prospective study incorporating these parameters would better explore the management outcome of this common condition.

\section{CONCLUSION}

At our centre, nasal bone fracture that needed reduction was frequently seen following physical assault in males predominently of 21 to 30 years. A combination of pain, epistaxis and nasal deformity was the commonest presentation. Reduction of fracture under local anesthesia within 16 days was the usual practice.

\section{CONFLICT OF INTEREST}

None declared.

\section{REFERENCES}

1. Mondin $V$, Rinaldo A, Ferlito A. Management of nasal bone fractures. Am J Otolaryngol. 2005 May 1;26(3):181-5.

2. Fernandes SV. Nasal fractures: the taming of the shrewd. Laryngoscope. 2004 Mar;114(3):587-92.

3. Rhee SC, Kim YK, Cha JH, Kang SR, Park HS. Septal fracture in simple nasal bone fracture. Plast Reconstr Surg. 2004 Jan 1;113(1):45-52.

4. Byun IH, Lee WJ, Roh TS, Hong JW. Demographic Factors of Nasal Bone Fractures and Social Reflection. J Craniofac Surg. 2020 Jan 1;31(1):16971.

5. Çil Y, Kahraman E. An analysis of 45 patients with pure nasal fractures. Ulus Travma Acil Cerrahi Derg. 2013 Mar 1;19(2):152-6.

6. Basheeth $N$, Donnelly M, David S, Munish S. Acute nasal fracture management: a prospective study and literature review. Laryngoscope. 2015 Dec;125(12):2677-84.

7. Watson DJ, Parker AJ, Slack RW, Griffiths MV. Local versus general anaesthetic in the management of the fractured nose. Clin Otolaryngol Allied Sci. 1988 Dec;13(6):491-4.

8. Kim J, Jung HJ, Shim WS. Corrective septorhinoplasty in acute nasal bone fractures. Clin Exp Otorhinolaryngol. 2018 Mar;11(1):46.

9. Hwang K, Ki SJ, Ko SH. Etiology of nasal bone fractures. J Craniofac Surg. 2017;28(3):785-8.

10. Wild DC, El Alami MA, Conboy PJ. Reduction of nasal fractures under local anaesthesia: an acceptable practice? The Surgeon. 2003 Feb 1;1(1):45-7. 
11. Al-Moraissi EA, Ellis III E. Local versus general anesthesia for the management of nasal bone fractures: a systematic review and meta-analysis. Journal of Oral and Maxillofacial Surgery. 2015 Apr 1;73(4):606-15.

12. Staffel JG. Optimizing treatment of nasal fractures. Laryngoscope. 2002 Oct;112(10):1709-19.

13. Konstantinidis I, Malliari $H$, Metaxas S. Nasal trauma: primary reconstruction with open rhinoplasty. Can J Plast Surg. 2011;19(3):108-10.

14. Ridder GJ, Boedeker CC, Fradis M, Schipper $\mathrm{J}$. Technique and timing for closed reduction of isolated nasal fractures: a retrospective study. Ear Nose Throat J. 2002 Jan;81(1):49-54.

15. Waldron J, Mitchell DB, Ford G. Reduction of fractured nasal bones; local versus general anaesthesia. Clin Otolaryngol Allied Sci. 1989 Aug;14(4):357-9. 